\title{
An Analytic Variational Study of the Mass Spectrum in $2+1$ Dimensional SU(3) Hamiltonian Lattice Gauge Theory
}

\author{
Jesse Carlsson; John A. L. McIntosh† Bruce H. J. McKellar \\ and Lloyd C. L. Hollenberg ${ }^{\S}$ \\ School of Physics, The University of Melbourne
}

November 14, 2018

\begin{abstract}
We calculate the masses of the lowest lying eigenstates of improved $\mathrm{SU}(2)$ and SU(3) lattice gauge theory in $2+1$ dimensions using an analytic variational approach. The ground state is approximated by a one plaquette trial state and mass gaps are calculated in the symmetric and antisymmetric sectors by minimising over a suitable basis of rectangular states.
\end{abstract}

\section{Introduction}

Lattice gauge theory (LGT) [1] is a non-perturbative technique used to regulate the ultraviolet divergences of non-abelian gauge theories. There are two standard formulations of LGT: the Lagrangian formulation and the Hamiltonian formulation.

To date most work on LGT has been carried out in the Lagrangian formulation for which Monte Carlo techniques are readily applicable. This approach involves replacing infinite and continuous space and time by a finite and discrete lattice of points. The Hamiltonian formulation developed by Kogut and Susskind [2] differs by leaving time continuous. In this framework the problem of gauge theory reduces to a many body problem and one is interested in calculating ground state and excited state configurations of the field defined on the lattice. The relation to continuum physics is realised in the limit in which the lattice spacing vanishes and the extent of the lattice becomes infinite.

Many body techniques, in particular plaquette expansion [3, 4, 5, 6] and the coupled cluster method [7, 8, 9, 10], have been applied to Hamiltonian LGT for a number of years. Not only do such calculations serve as a check on the more common Monte Carlo techniques of the Lagrangian approach, they also provide more immediate access to excited

\footnotetext{
*e-mail:j.carlsson@physics. unimelb.edu .au

${ }^{\dagger}$ e-mail:jam@physics . unimelb.EDU.AU

${ }^{\ddagger}$ e-mail:b.mckellar@physics . unimelb.edu . au

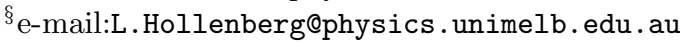


states [11. In addition, the problem of finite density QCD may be more easily handled in the Hamiltonian approach [12].

In recent years an improvement program has been undertaken in the Lagrangian approach. The aim has been to systematically correct the discretisation errors arising in the lattice Lagrangian and hence decrease the computational cost of lattice calculations [13]. More recently this improvement program has moved to the Hamiltonian formulation [14]. In a recent paper we demonstrated how improved Hamiltonians can be constructed [15]. In this paper we explore the use of improved Hamiltonians in variational calculations of $\mathrm{SU}(2)$ and $\mathrm{SU}(3)$ vacuum energy densities, specific heats and exited state energies on a $2+1$ dimensional lattice. A key development in this paper is the application of analytic techniques to $\mathrm{SU}(3) \mathrm{LGT}$.

The outline of this paper is as follows. In section 2 we introduce a generating function for $\mathrm{SU}(3)$ which allows us perform analytic calculations of energy densities and specific heats. In section 3 we calculate the energies of the lowest lying eigenstates of SU(2) and $\mathrm{SU}(3)$ pure gauge theory in $2+1$ dimensions and compare the results of unimproved and improved calculations. Section 4 contains our conclusions.

\section{Vacuum Energy Density and Specific Heat}

\section{$2.1 \quad$ Introduction}

In this section we calculate vacuum energy densities and specific heats for the Kogut Susskind, improved and tadpole improved Hamiltonians. Improved Hamiltonians are easily constructed by adding appropriately weighted gauge invariant terms to the Kogut Susskind Hamiltonian [15]. For SU $(N)$ pure gauge theory with coupling $g^{2}$ on a lattice with spacing $a$, we define a class of Hamiltonians in terms of the link variables $U_{j}(\boldsymbol{x})$ and electric fields $E_{i}(\boldsymbol{x})$ (which are themselves defined in the appendix), and two parameters $\kappa$ and $u_{0}$ which define the particular Hamiltonian in question:

$$
\begin{aligned}
\tilde{H}\left(\kappa, u_{0}\right)= & \frac{g^{2}}{a} \sum_{\boldsymbol{x}, i} \operatorname{Tr}\left[(1-\kappa) E_{i}(\boldsymbol{x})^{2}+\frac{\kappa}{u_{0}^{2}} E_{i}(\boldsymbol{x}) U_{i}(\boldsymbol{x}) E_{i}(\boldsymbol{x}+a \boldsymbol{i}) U_{i}^{\dagger}(\boldsymbol{x})\right] \\
& +\frac{2 N}{a g^{2}} \sum_{\boldsymbol{x}, i<j}\left\{(1+4 \kappa) P_{i j}(\boldsymbol{x})-\frac{\kappa}{2}\left[R_{i j}(\boldsymbol{x})+R_{j i}(\boldsymbol{x})\right]\right\},
\end{aligned}
$$

where

$$
\begin{aligned}
P_{i j}(\boldsymbol{x}) & =1-\frac{1}{N u_{0}^{4}} \operatorname{Re} \operatorname{Tr}\left[U_{i}(\boldsymbol{x}) U_{j}(\boldsymbol{x}+\boldsymbol{i} a) U_{i}^{\dagger}(\boldsymbol{x}+\boldsymbol{j} a) U_{j}^{\dagger}(\boldsymbol{x})\right] \\
& \equiv 1-\frac{1}{N u_{0}^{4}} \operatorname{Re}(\square)
\end{aligned}
$$

and

$$
R_{i j}(x)=1-\frac{1}{N u_{0}^{6}} \operatorname{Re}(\breve{\square})
$$


are the plaquette and rectangle operators respectively. The Kogut-Susskind and $\mathcal{O}\left(a^{2}\right)$ classically improved Hamiltonians are given by $H_{K S}=\tilde{H}(0,1)$ and $H_{I}=\tilde{H}(1 / 6,1)$ respectively. The tadpole improved Hamiltonian is given by $H_{T I}=\tilde{H}\left(1 / 6, u_{0}\right)$, where $u_{0}$ is now the mean link, defined by

$$
u_{0}^{4}=\frac{1}{N}\langle\sqcup\rangle
$$

and evaluated self-consistently, as a function of $g^{2}$. Details of the mean link calculation are given in section 2.3 .

We approximate the ground, or (perturbed) vacuum, state, with energy $E_{0}$, with the one plaquette trial state, first suggested by Greensite [16],

$$
\left|\phi_{0}\right\rangle=\exp \left[\frac{c}{2} \sum_{p}\left(Z_{p}+\bar{Z}_{p}\right)\right]|0\rangle .
$$

Here, $|0\rangle$ is the strong coupling vacuum defined by $E_{i}^{\alpha}(\boldsymbol{x})|0\rangle=0$ for all $i, \boldsymbol{x}$ and $\alpha=$ $1,2, \ldots, N^{2}-1$. The $p$-sum extends over all plaquettes on the lattice and

$$
Z_{p}=\operatorname{Tr}\left[U_{i}(\boldsymbol{x}) U_{j}(\boldsymbol{x}+\boldsymbol{i} a) U_{i}^{\dagger}(\boldsymbol{x}+\boldsymbol{j} a) U_{j}^{\dagger}(\boldsymbol{x})\right],
$$

denotes the plaquette labelled by $p$ which joins the lattice sites $\boldsymbol{x}, \boldsymbol{x}+\boldsymbol{i} a, \boldsymbol{x}+(\boldsymbol{i}+\boldsymbol{j}) a$ and $\boldsymbol{x}+\boldsymbol{j} a . \quad \bar{Z}_{p}$ is the trace of the link operators in the opposite direction. For a given coupling the variational parameter $c$ is fixed by minimising the vacuum energy density, which, after some algebra making use of eqn(47) and eqns (55 59) from the appendix, can be expressed in terms of the expectation values of plaquettes and rectangles as follows:

$$
\begin{aligned}
\epsilon_{0} & =\frac{a}{N_{p}}\langle\tilde{H}\rangle \\
& =\left[(1-\kappa)\left(\frac{N^{2}-1}{2 \beta}\right) c-\frac{2 \beta(1+4 \kappa)}{N u_{0}^{4}}\right]\langle\square\rangle+\frac{2 \kappa \beta}{N u_{0}^{6}}\langle\square\rangle
\end{aligned}
$$

Here $N_{p}$ is the number of plaquettes on the lattice and $\beta=N / g^{2}$ is the inverse coupling.

\subsection{Analytic Techniques for SU(2) and SU(3)}

The expression for the $\mathrm{SU}(N)$ vaccuum energy density given by eqn(尹) allows us, in principle, to fix the variational parameter $c$ for a given $\beta$ in any number of dimensions. The difficulty lies in the calculation of the plaquette and rectangle expectation values. Monte Carlo simulations can be used to calculate these expectation values in any number of dimensions. However, for the special case of $2+1$ dimensions, the calculation can be carried out analytically. This is because the change of variables from links to plaquettes has unit Jacobian [17. Consequently, the plaquettes on the two dimensional lattice are independent variables, which leads to:

$$
\langle\sqcup\rangle=\frac{\int \prod_{l} d U_{l} Z_{0} \prod_{p} e^{c\left(Z_{p}+\bar{Z}_{p}\right)}}{\int \prod_{l} d U_{l} \prod_{p} e^{c\left(Z_{p}+\bar{Z}_{p}\right)}}=\frac{\int d V \operatorname{Tr} V e^{c \operatorname{Tr}\left(V+V^{\dagger}\right)}}{\int d V e^{c \operatorname{Tr}\left(V+V^{\dagger}\right)}}
$$


and

$$
\langle\hookrightarrow\rangle=\frac{1}{N}\langle\sqcup\rangle^{2}
$$

Eqn(9) makes use of the independence of plaquettes and eqn(29). For the case of SU(2), analytic expressions for the plaquette expectation value in terms of modified Bessel functions $\left(I_{n}\right)$ have been used in variational calculations for the last 20 years:

$$
\langle\square\rangle=\frac{2 I_{2}(4 c)}{I_{1}(4 c)} \text {. }
$$

We derived the corresponding $\mathrm{SU}(3)$ result, which has not been used, to our knowledge, in the Hamiltonian approach, and then discovered that it follows simply from a paper of Eriksson et al [18], in which the SU(3) integral,

$$
\int d U e^{\operatorname{Tr}\left(U M^{\dagger}+U^{\dagger} M\right)}
$$

is calculated for arbitrary $3 \times 3$ matrices $M$. Following their analysis, but treating $M$ and $M^{\dagger}$ as independent variables, with $M=c \mathbb{1}$ and $M^{\dagger}=d 1$, leads to an expression for the $\mathrm{SU}(3)$ generating function:

$$
\begin{aligned}
\mathcal{Y}(c, d) & =\int d U e^{\operatorname{Tr}\left(c U+d U^{\dagger}\right)} \\
& =\frac{i}{\pi} \oint_{\Gamma} d z \frac{e^{-\left(c^{3}+d^{3}\right) / z}}{z(z-c d)^{3 / 2}} J_{1}\left(\frac{2}{z}(z-c d)^{3 / 2}\right) .
\end{aligned}
$$

Here $J_{1}$ is the first Bessel function and $\Gamma$ is a closed contour in the complex plane including the pole at $z=0$ but excluding the pole at $z=c d$. To evaluate this contour integral we expand the integrand in power series about the pole at $z=0$ and use Cauchy's integral theorem to eventually obtain,

$$
\mathcal{Y}(c, d)=2 \sum_{k=0}^{\infty} \frac{1}{(k+1) !(k+2) !} \sum_{l=0}^{k}\left(\begin{array}{c}
3 k+3 \\
k-l
\end{array}\right) \frac{1}{l !}(c d)^{k-l}\left(c^{3}+d^{3}\right)^{l} .
$$

This generating functional is extremely useful for Hamiltonian lattice calculations?. In principle, it allows us to investigate $2+1$ dimensional pure $\mathrm{SU}(3)$ gauge theory analytically. The calculation of various matrix elements for all couplings, reduces to a exercise in differentiation. For example:

$$
\begin{aligned}
\left\langle Z_{p}\right\rangle & \equiv\langle\square\rangle=\left[\frac{1}{\mathcal{Y}} \frac{\partial \mathcal{Y}}{\partial c}\right]_{d=c}, \\
\left\langle Z_{p} \bar{Z}_{p}\right\rangle & \equiv\left\langle\frac{1}{\mathcal{Y}} \frac{\partial^{2} \mathcal{Y}}{\partial c \partial d}\right]_{d=c}, \text { and } \\
\left\langle Z_{p}^{m} \bar{Z}_{p}^{n}\right\rangle & =\left[\frac{1}{\mathcal{Y}} \frac{\partial^{m+n} \mathcal{Y}}{\partial c^{m} \partial d^{n}}\right]_{d=c} .
\end{aligned}
$$

\footnotetext{
${ }^{1} \mathrm{The} \mathrm{SU}(N)$ generalisation of this result, which we have recently obtained, will be reported in a future publication.
} 


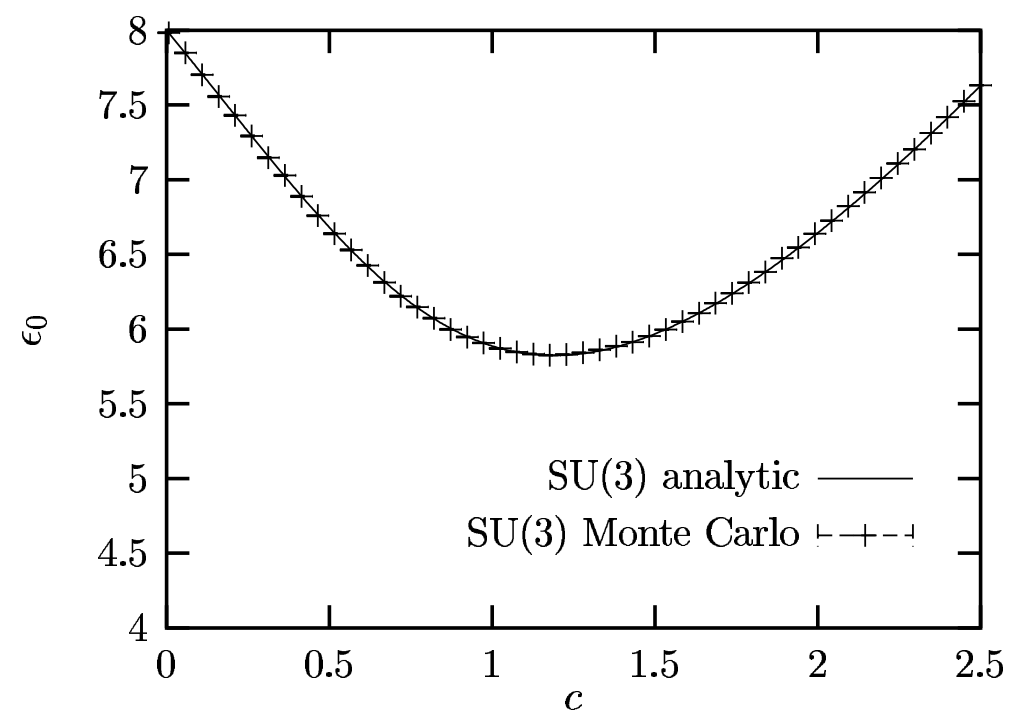

Figure 1: Analytic and Monte Carlo calculations of the SU(3) vacuum energy density in $2+1$ dimensions for $\beta=4$.0. It should be noted that the errors in the Monte Carlo calculation are smaller than the plot points.

As a check on this series we calculate the strong coupling limit $(c=0)$ of $\left\langle Z_{p}^{m} \bar{Z}_{p}^{n}\right\rangle$. By differentiating eqn(13), we observe that the only non-zero strong coupling matrix elements occur when $n+2 m \equiv 0 \bmod 3$. For this case we have,

$$
\left\langle Z_{p}^{m} \bar{Z}_{p}^{n}\right\rangle_{c=0}=\sum_{k}\left(\begin{array}{c}
3 k+3 \\
n+m+3
\end{array}\right) \frac{2 n ! m !}{(k+1) !(k+2) !\left(\frac{n+2 m}{3}-k\right) !\left(\frac{m+2 n}{3}-k\right) !} .
$$

Here the sum runs over all integers $\frac{n+m}{3} \leq k \leq \min \left(\frac{n+2 m}{3}, \frac{m+2 n}{3}\right)$. This strong coupling result has an equivalent combinatoric interpretation as the number of times the singlet representation appears in the direct product of $m 3$ and $n \overline{3}$ representations [19]. A simple Mathematica code verifies the agreement of the two approaches.

A demonstration of the accuracy of the series away from the strong coupling limit is provided by a Monte Carlo calculation of the vacuum energy density at various couplings. The example of $\beta=4.0$ is shown in fig 1 and indicates close agreement between the Monte Carlo and analytic calculations.

\section{$2.3 \quad$ Results}

To calculate the energy density and variational parameter as functions of $\beta$ we proceed as follows. For the Kogut-Susskind and classically improved cases, we simply minimise $\epsilon_{0}$ for a given value of $\beta$. The value of $c$ at which $\epsilon_{0}$ takes its minimum defines $c$ as a function of $\beta$.

The tadpole improved calculation is more complicated. This is because the mean plaquette, from which $u_{0}$ is calculated, depends on the variational state $\left|\phi_{0}\right\rangle$. To complicate matters, the variational state in turn depends on the energy density and hence $u_{0}$. Such interdependence suggests the use of the following iterative approach. For a given $\beta$ and 


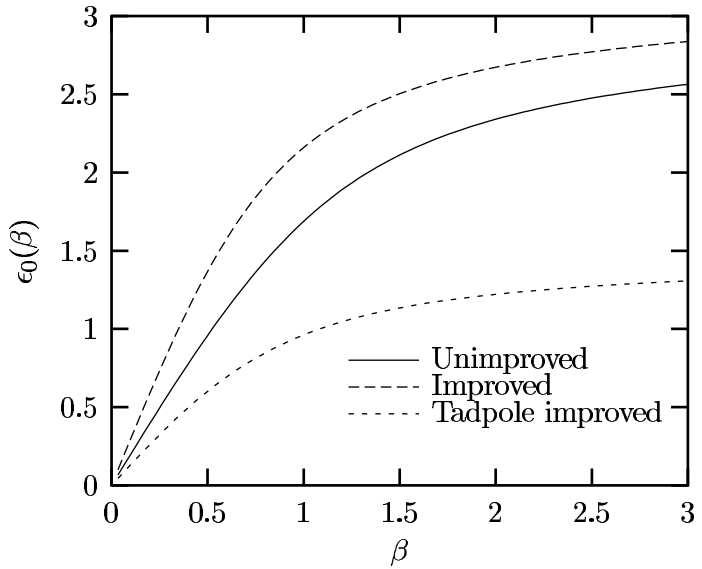

(a) $\mathrm{SU}(2)$

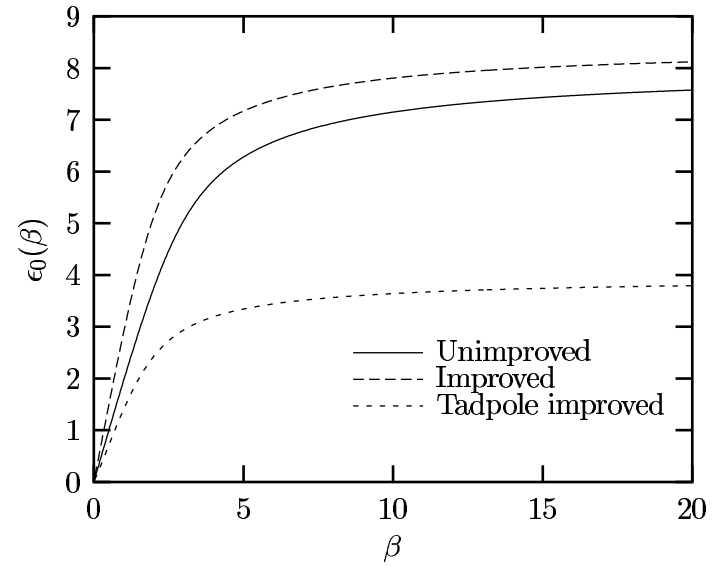

(b) $\mathrm{SU}(3)$

Figure 2: Analytic calculation of the unimproved, improved and tadpole improved vacuum energy density in $2+1$ dimensions for $\mathrm{SU}(2)$ and $\mathrm{SU}(3)$.

initial approximation of $u_{0}$ we minimise the energy density to fix the variational state $\left|\phi_{0}\right\rangle$. Using this trial state, we update $u_{0}$ for use in the next iteration. This process is iterated until convergence is achieved, typically after only a few iterations.

The results of the Kogut-Susskind, classically improved and tadpole improved SU(2) and $\mathrm{SU}(3)$ vacuum energy density calculations are shown in fig 2. The corresponding variational parameters $c(\beta)$ are shown in fig 3. The correct strong and weak coupling behavior is observed in each case. The differing gradients in the weak coupling limit highlight the fact that when using an improved Hamiltonian one is using a different renormalisation scheme to the unimproved case.

In practice the $\mathrm{SU}(3)$ generating function is truncated. The dependence of the variational parameter on various truncations of the $k$-sum in eqn $(13)$ is shown in fig 4 . We see that convergence is achieved up to $\beta \approx 13$ when keeping 20 terms. Further calculations show that when keeping 50 terms convergence up to $\beta \approx 30$ is achieved.

In addition to the vacuum energy density we can also calculate the specific heat:

$$
C_{V}=-\frac{\partial^{2} \epsilon_{0}}{\partial \beta^{2}}
$$

The results are shown in fig 5 . We note that the location of the peak indicates the region of transition from strong to weak coupling. For an improved calculation one would expect the peak to be located at a smaller $\beta$ (corresponding to a larger coupling) than for the equivalent unimproved calculation. We see that this is indeed the case for both $\mathrm{SU}(2)$ and $\mathrm{SU}(3)$, with the tadpole improved Hamiltonian demonstrating the largest degree of improvement. 


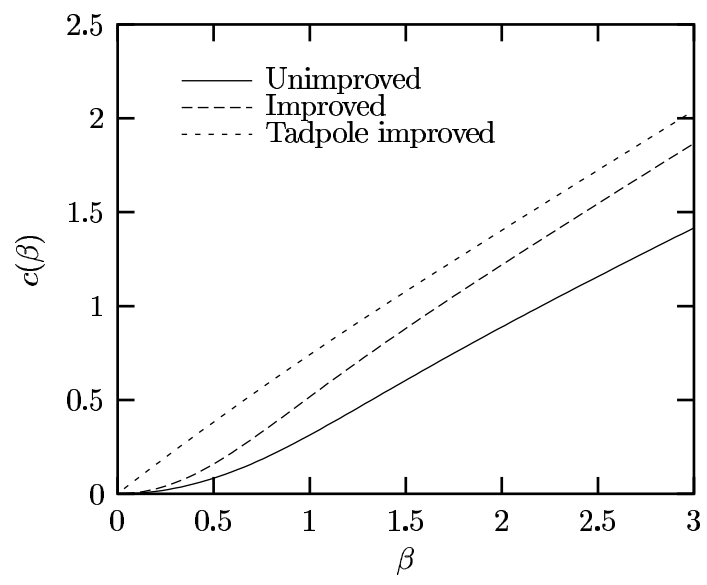

(a) $\mathrm{SU}(2)$

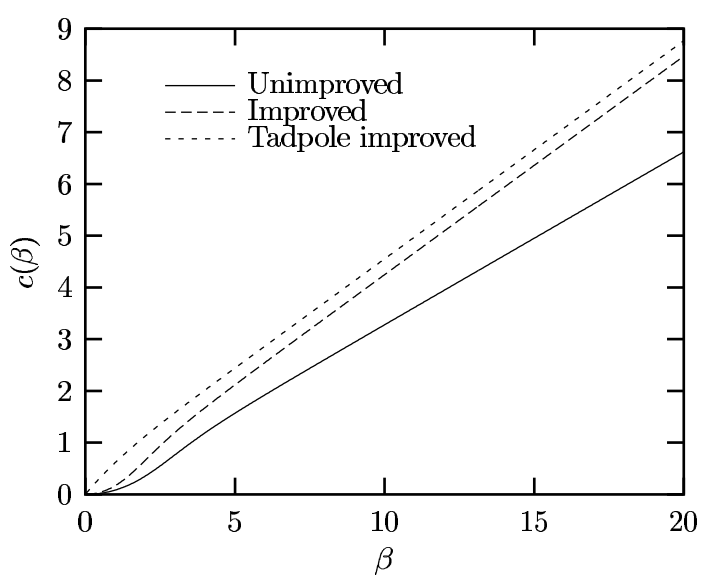

(b) $\mathrm{SU}(3)$

Figure 3: Analytic calculation of the unimproved, improved and tadpole improved variational parameter in $2+1$ dimensions for $\mathrm{SU}(2)$ and $\mathrm{SU}(3)$.

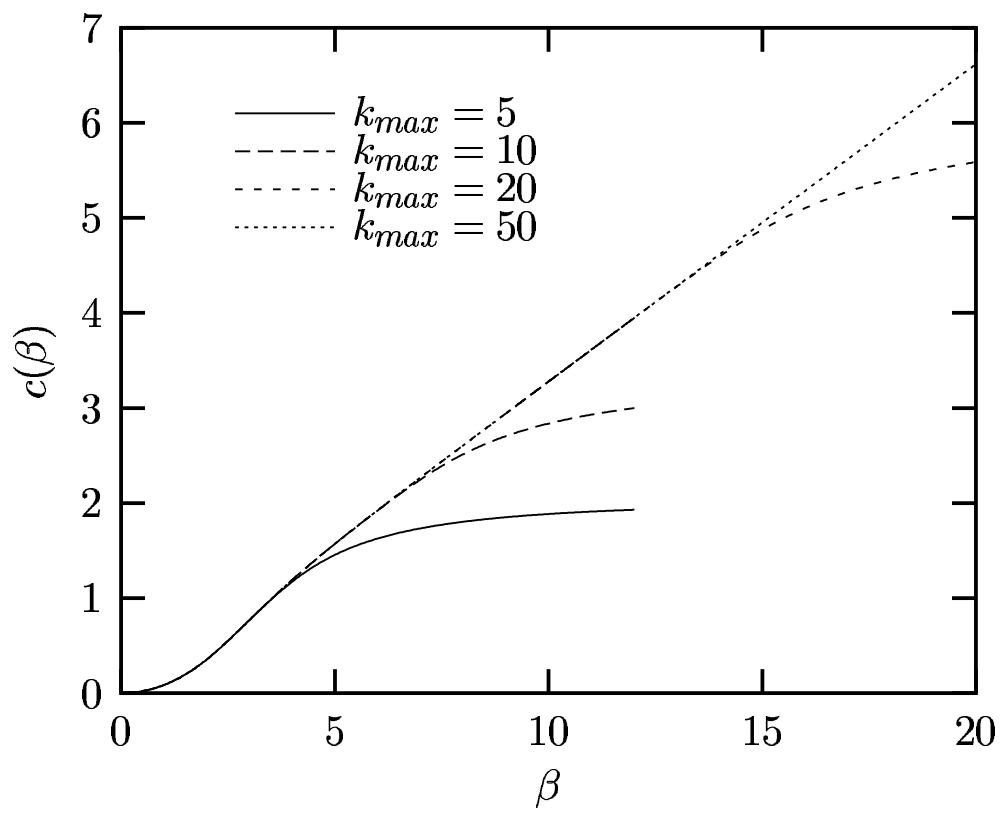

Figure 4: Analytic calculation of the unimproved SU(3) variational parameter in $2+1$ dimensions, truncating the $k$-sum of $\mathcal{Y}(c, d)$ at $k=k_{\text {max }}$. 


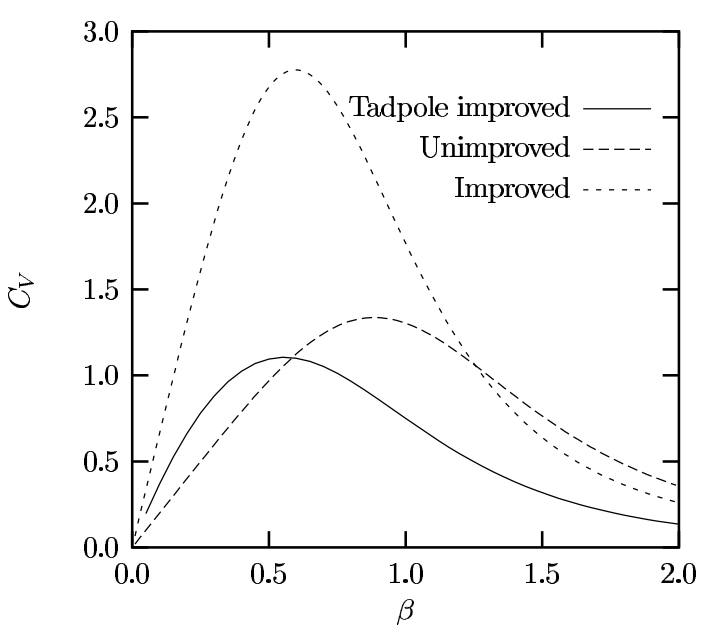

(a) $\mathrm{SU}(2)$

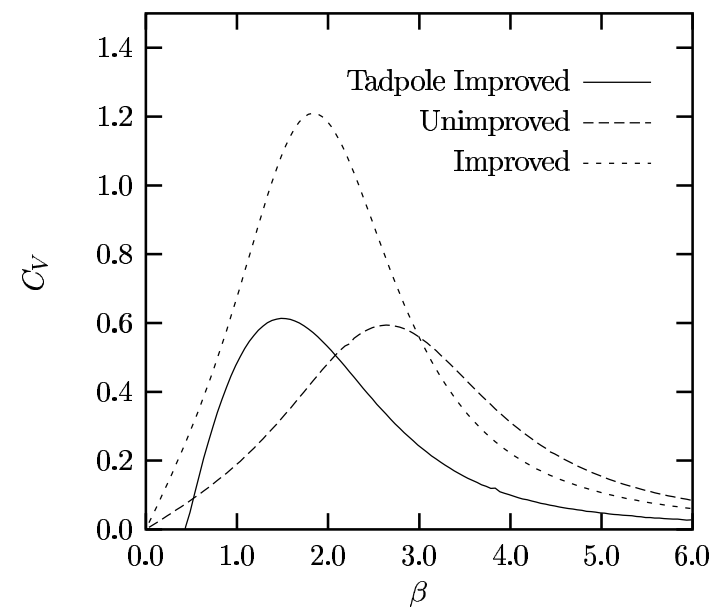

(b) $\mathrm{SU}(3)$

Figure 5: The unimproved, improved and tadpole improved specific heat in $2+1$ dimensions for $\mathrm{SU}(2)$ and $\mathrm{SU}(3)$.

\section{Mass Gaps}

\subsection{Introduction}

Having fixed the chosen trial vacuum $\left|\phi_{0}\right\rangle$, in this section we turn to investigating excited states. Our aim is to calculate the lowest lying energy eigenstates of the Hamiltonians described by eqn(11) for both $\mathrm{SU}(2)$ and $\mathrm{SU}(3)$.

We follow Arisue [20] and expand the excited state $\left|\phi_{1}\right\rangle$ in the basis consisting of all rectangular Wilson loops $\{|l\rangle\}_{l=1}^{L_{\max }}$ that fit in a given square whose side length $L_{\max }$ defines the order of the calculation. In order to ensure the orthogonality of $\left|\phi_{0}\right\rangle$ and $\left|\phi_{1}\right\rangle$ we parameterise the excited state as follows

$$
\left|\phi_{1}\right\rangle=\sum_{n, m=1}^{L_{\max }} s_{l}|n, m\rangle=\sum_{l=1}^{L_{\max }^{2}} s_{l}|l\rangle,
$$

with,

$$
|l\rangle=\sum_{\boldsymbol{x}}\left[W_{l}(\boldsymbol{x})-\left\langle W_{l}(\boldsymbol{x})\right\rangle\right]\left|\phi_{0}\right\rangle
$$

Here $\left\langle W_{l}(\boldsymbol{x})\right\rangle$ is the expectation value of $W_{l}(\boldsymbol{x})$ with respect to the ground state $\left|\phi_{0}\right\rangle$ and the convenient label $l=(n-1) L_{\max }+m$ has been defined to label the $n \times m$ rectangular state. We define the particular form of $W_{l}(\boldsymbol{x})$ to reflect the symmetry of the sector we wish to consider. For SU(3) we take $W_{l}(\boldsymbol{x})=\operatorname{Tr}\left[w_{l}(\boldsymbol{x}) \pm w_{l}^{\dagger}(\boldsymbol{x})\right]$ for the symmetric sector and antisymmetric sector. To avoid over-decorated equations, the particular $W_{l}(\boldsymbol{x})$ in use is to be deduced from the context. Here $w_{l}(\boldsymbol{x})$ is the rectangular Wilson loop joining the 
lattice sites $\boldsymbol{x}, \boldsymbol{x}+n a \boldsymbol{i}, \boldsymbol{x}+n a \boldsymbol{i}+$ maj and $\boldsymbol{x}+$ naj, with

$$
n=\left[\frac{l-1}{L_{\max }}\right]+1 \quad \text { and } \quad m=l-L_{\max }\left[\frac{l-1}{L_{\max }}\right] .
$$

Here $[k]$ denotes the integer part of $k$. In order to calculate excited state energies we minimise the mass gap (the difference between the excited state and ground state energy) over the basis defined by a particular order $L_{\max }$. To do this we again follow Arisue [20] and define the matrices

$$
N_{l l^{\prime}}=\frac{1}{N_{p}}\left\langle l\left|\tilde{H}-E_{0}\right| l^{\prime}\right\rangle
$$

and

$$
D_{l l^{\prime}}=\frac{1}{N_{p}}\left\langle l \mid l^{\prime}\right\rangle=\sum_{\boldsymbol{x}}\left[\left\langle W_{l}^{\dagger}(\boldsymbol{x}) W_{l^{\prime}}(\mathbf{0})\right\rangle-\left\langle W_{l}(\boldsymbol{x})\right\rangle^{*}\left\langle W_{l^{\prime}}(\mathbf{0})\right\rangle\right]
$$

Extending the calculation to the general improved Hamiltonian $\tilde{H}\left(\kappa, u_{0}\right)$ and making use of eqns 56 59 from the appendix gives

$$
\begin{aligned}
N_{l l^{\prime}}=-\frac{g^{2}}{2 a} \sum_{i, \boldsymbol{x}} \sum_{\boldsymbol{x}^{\prime}} & \left\{(1-\kappa)\left\langle\left[E_{i}^{\alpha}(\boldsymbol{x}), W_{l}^{\dagger}\left(\boldsymbol{x}^{\prime}\right)\right]\left[E_{i}^{\alpha}(\boldsymbol{x}), W_{l^{\prime}}(\mathbf{0})\right]\right\rangle\right. \\
+ & \left.\frac{\kappa}{u_{0}^{2}}\left\langle\left[E_{i}^{\alpha}(\boldsymbol{x}), W_{l}^{\dagger}\left(\boldsymbol{x}^{\prime}\right)\right]\left[\tilde{E}_{i}^{\alpha}(\boldsymbol{x}+a \boldsymbol{i}), W_{l^{\prime}}(\mathbf{0})\right]\right\rangle\right\} .
\end{aligned}
$$

To minimise the mass gap over a basis of a given order we make use of following diagonalisation technique 21]. We first diagonalise $D$, with

$$
S^{\dagger} D S=V^{2}
$$

where $V$ is diagonal. The $n$-th lowest eigenvalue of the modified Hamiltonian

$$
H^{\prime}=V S^{\dagger} N S V
$$

then gives an upper bound for the mass gap corresponding to the $n$-th lowest eigenvalue of the Hamiltonian, $\Delta m_{n}$.

\subsection{Calculating Matrix Elements}

Having described the minimisation process we now detail the calculation of the matrix elements $N_{l l^{\prime}}$ and $D_{l l^{\prime}}$. Our aim is to reduce $N_{l l^{\prime}}$ and $D_{l l^{\prime}}$ to polynomials of one plaquette matrix elements. This, again has been done for the case of $\mathrm{SU}(2)$ by Arisue [20]. Here we retrace his calculations for the case of $\mathrm{SU}(3)$ and extend them to incorporate improved Hamiltonians. We start with $D_{l l^{\prime}}$.

Taking elementary plaquettes as our independent variables, it is easy to show that the only non-zero contributions to $D_{l l^{\prime}}$ occur when the rectangles $l$ and $l^{\prime}$ overlap. As an example of a contribution to $D_{l l^{\prime}}$, consider $\Delta D_{l l^{\prime}}$; the case where $N_{l \cap l^{\prime}}$ plaquettes are contained by 
both rectangles (these are the overlap plaquettes) and $N_{l}$ plaquettes are contained by the rectangle $l$. In order to calculate such matrix elements we rely heavily on the orthogonality properties of $\mathrm{SU}(N)$ characters. We are interested in calculating $\mathrm{SU}(N)$ integrals of the form

$$
\int d U_{p} e^{S\left(U_{p}\right)} \chi_{r}\left(U_{p} V\right)
$$

where $U_{p}$ is a $\mathrm{SU}(N)$ plaquette variable and $V$ is a product of any number of plaquettes not including $U_{p}$. Here $\chi_{r}(U)$ denotes the character corresponding to the representation $r$. For $\mathrm{SU}(2), r=0,1 / 2,3 / 2, \ldots$ and for $\mathrm{SU}(3), r=(\lambda, \mu)$ where $\lambda$ denotes the number of boxes in the first row of the Young's Tableaux describing the representation and $\mu$ is the number of boxes in the second row. Similarly, for $\mathrm{SU}(N), r=\left(r_{1}, r_{2}, \ldots, r_{N-1}\right)$.

Performing a character expansion of the exponent in eq. 25 gives:

$$
\int d U_{p} e^{S\left(U_{p}\right)} \chi_{r}\left(U_{p} V\right)=\sum_{r^{\prime}} \int d U_{p} c_{r^{\prime}} \chi_{r^{\prime}}\left(U_{p}\right) \chi_{r}\left(U_{p} V\right)
$$

This is simply a generalisation of a Fourier expansion. Here, the coefficient $c_{r^{\prime}}$ is given by:

$$
c_{r^{\prime}}=\int d U_{p} \chi_{r^{\prime}}\left(U_{p}\right) e^{S\left(U_{p}\right)} .
$$

Now using the orthogonality relation,

$$
\int d U_{p} \chi_{r^{\prime}}\left(U_{p} V\right) \chi_{r}\left(U_{p}\right)=\frac{1}{d_{r}} \delta_{r^{\prime} r} \chi_{r}(V)
$$

where $d_{r}$ is the dimension of the representation $r$, we obtain:

$$
\int d U_{p} e^{S\left(U_{p}\right)} \chi_{r}\left(U_{p} V\right)=\frac{1}{d_{r}} \chi_{r}(V) \int d U_{p} \chi_{r}\left(U_{p}\right) e^{S\left(U_{p}\right)} .
$$

This result allows us to integrate out a single plaquette from an extended Wilson loop in a given representation $r$. To complete the calculation we need to relate characters to traces of group elements. This can be done using Weyl's character formula [22]. For SU(3) the dimensions and characters corresponding to the first few representations are given by:

$$
\begin{array}{rlrl}
\chi_{10}(U) & =\operatorname{Tr} U & d_{10}=3 \\
\chi_{11}(U) & =\frac{1}{2}\left[(\operatorname{Tr} U)^{2}-\operatorname{Tr}\left(U^{2}\right)\right]=\operatorname{Tr} U^{\dagger} & d_{11}=3 \\
\chi_{20}(U)=\frac{1}{2}\left[(\operatorname{Tr} U)^{2}+\operatorname{Tr}\left(U^{2}\right)\right]=(\operatorname{Tr} U)^{2}-\operatorname{Tr} U^{\dagger} & d_{20}=6 \\
\chi_{21}(U)=\frac{1}{3}\left[(\operatorname{Tr} U)^{3}-\operatorname{Tr}\left(U^{3}\right)\right]=\operatorname{Tr} U \operatorname{Tr} U^{\dagger}-1 & d_{21}=8
\end{array}
$$

Here we have made use of the SU(3) identity

$$
\operatorname{Tr}\left(U^{2}\right)=(\operatorname{Tr} U)^{2}-2 \operatorname{Tr} U^{\dagger} .
$$

Applying eqns 29 21) allows us to obtain analytic expressions for each contribution to $D_{l l^{\prime}}$. For the case of $\Delta D_{l l^{\prime}}$ described earlier, we have

$$
\Delta D_{l l^{\prime}}=\frac{2}{3} F_{Z}\left(N_{l}+N_{l^{\prime}}-2 N_{l \cap l^{\prime}}\right)\left[F_{Z^{2}}\left(N_{l \cap l^{\prime}}\right)+F_{Z \bar{Z}}\left(N_{l \cap l^{\prime}}\right)\right]-4 F_{Z}\left(N_{l}\right) F_{Z}\left(N_{l^{\prime}}\right),
$$


where the character integrals are given by:

$$
\begin{aligned}
F_{Z}(n) & =\left(\frac{1}{3}\right)^{n-1}\left\langle Z_{p}\right\rangle^{n}, \\
F_{Z^{2}}(n) & =\left(\frac{1}{6}\right)^{n-1}\left\langle Z_{p}^{2}-\bar{Z}_{p}\right\rangle^{n}+\left(\frac{1}{3}\right)^{n-1}\left\langle\bar{Z}_{p}\right\rangle^{n}, \text { and } \\
F_{Z \bar{Z}}(n) & =1+\left(\frac{1}{8}\right)^{n-1}\left(\left\langle Z_{p} \bar{Z}_{p}\right\rangle-1\right)^{n} .
\end{aligned}
$$

We now move on to the calculation of $N_{l l^{\prime}}$. It is easy to show that the only non-zero contributions occur when there is at least one common link and an overlap between the rectangles. The improvement term (the second term in eqn(22)) only contributes when the two rectangles share at least two links in a given direction. Consider the contribution $\Delta N_{l l^{\prime}}$ to $N_{l l^{\prime}}$ in which there are $L_{1}$ common links and $L_{2}$ common strings of two links in a given direction. Again we suppose $N_{l}$ plaquettes are enclosed by rectangle $l$ and that there are $N_{l \cap l^{\prime}}$ common plaquettes. Making use of eqn(29) and eqns(56 59) from the appendix we obtain

$$
\Delta N_{l l^{\prime}}=\frac{L}{3} F_{Z}\left(N_{l}+N_{l^{\prime}}-2 N_{l \cap l^{\prime}}\right)\left[\frac{2}{3} F_{Z^{2}}\left(N_{l \cap l^{\prime}}\right)-2 F_{Z}\left(N_{l \cap l^{\prime}}\right)-3+\frac{1}{3} F_{Z \bar{Z}}\left(N_{l \cap l^{\prime}}\right)\right],
$$

with

$$
L=(1-\kappa) L_{1}+\frac{\kappa}{u_{0}^{2}} L_{2}
$$

Having calculated individual contributions to $D_{l l^{\prime}}$ and $N_{l l^{\prime}}$, the completion of the calculation requires counting the possible overlaps of a given type. The approach described in this section is easily extended to $\mathrm{SU}(N)$. However, analytic expressions for the character integrals are not available for this case.

\subsection{Results}

For each SU(3) calculation we have kept 80 terms in the $k$-sum of eqn(13) giving convergence up to $\beta=50$. The generation of $N_{l l^{\prime}}$ and $D_{l l^{\prime}}$ and implementation of the minimisation process was accomplished with a Mathematica code.

For the case of $2+1$ dimensions we expect $\Delta m / g^{2}$ to become constant in the scaling region. The convergence of the massgaps with $L_{\max }$ for the cases of $\mathrm{SU}(2)$ and $\mathrm{SU}(3)$ is illustrated in figs 6(a) and 6(b). We notice that for the case of SU(3) only small improvements to scaling are gained by extending the calculation beyond order 8 . This suggests that a more complicated basis (including, for example, loops covered more than once) is required to simulate $\mathrm{SU}(3)$ excited states than for the case of $\mathrm{SU}(2)$.

From fig 7, we see that $\Delta m_{1}^{S} / g^{2}$ is approximated well by a constant in very large scaling regions for the lowest lying eigenstates for both $\mathrm{SU}(2)$ and $\mathrm{SU}(3)$. The scaling behavior becomes significantly worse for the antisymmetric sector and for higher energy eigenvalues. This is because the simplistic form of our excited state wave function is not sufficient to reproduce the plaquette correlations required to simulate these higher order states. One 


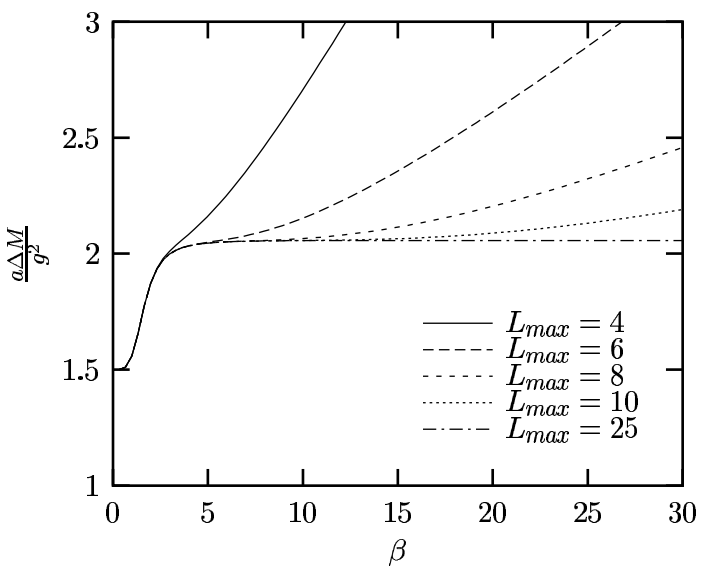

(a) $\mathrm{SU}(2)$

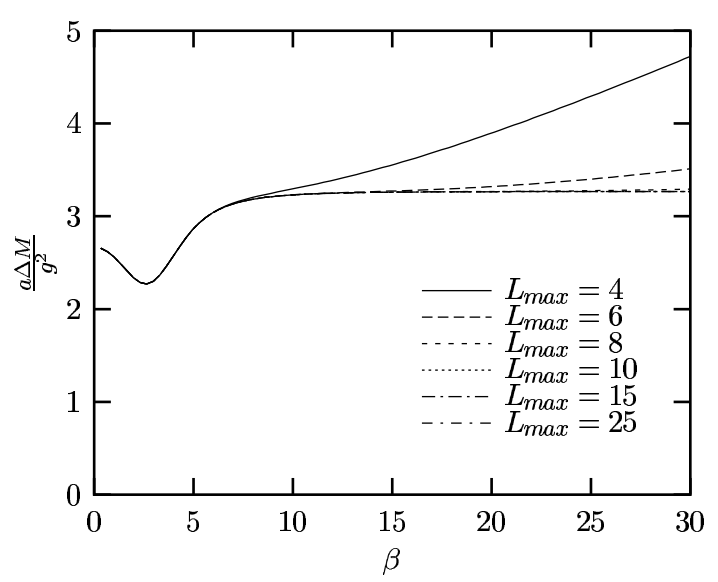

(b) $\mathrm{SU}(3)$

Figure 6: The unimproved 2+1 dimensional symmetric massgaps for $\mathrm{SU}(2)$ and $\mathrm{SU}(3)$.

would expect the simulation of higher order eigenstates to improve by including more complicated loops in our expansion basis or by using a more complicated ground state. The continuum limit results of excited states for $\mathrm{SU}(2)$ and $\mathrm{SU}(3)$ results are given in tables 1 - 3.

For the unimproved $\mathrm{SU}(2)$ case, the lowest two eigenstates agree precisely with the calculation of Arisue [20]. This serves as a check on our counting in calculating the possible overlaps of excited states. Our calculation is in disagreement with that of Arisue at the 3rd eigenstate. It appears that our 4th eigenstate corresponds to Arisue's 3rd and that our third eigenstate does not appear in his results. The reasons for this are not clear.

The results for the $\mathrm{SU}(3)$ symmetric massgap (in units of $g^{2}$ ) are to be compared to calculations by Luo and Chen 2.15 \pm 0.06 [23], Samuel 1.84 \pm 0.46 [24] and Teper 2.40 \pm 0.02 [25]. It should be stressed that although our result of $3.26520 \pm 0.00009$ is considerably higher, it provides a precise upper upper-bound on the exact result. By including more complicated loops in the expansion basis one would expect to reduce this upper bound. This is emphasised by the fact that when using only square basis states the result is considerably higher.

When compared to equivalent unimproved calculations, the improved and tadpole improved massgaps approach scaling faster as $\beta$ is increased. This is evident in figs 6 and 7 and is expected since, for an improved calculation one is closer to the continuum limit when working at a given coupling. However, for each improved calculation the scaling behaviour is not as precise as the equivalent unimproved calculation. This is clear from tables 1 3. A possible reason for this is that the one plaquette trial state used here does not allow for direct contributions from the improvement term in the kinetic Hamiltonian. For this term to contribute directly one would need a trial state which includes Wilson loops extending at least two links in at least one direction. 


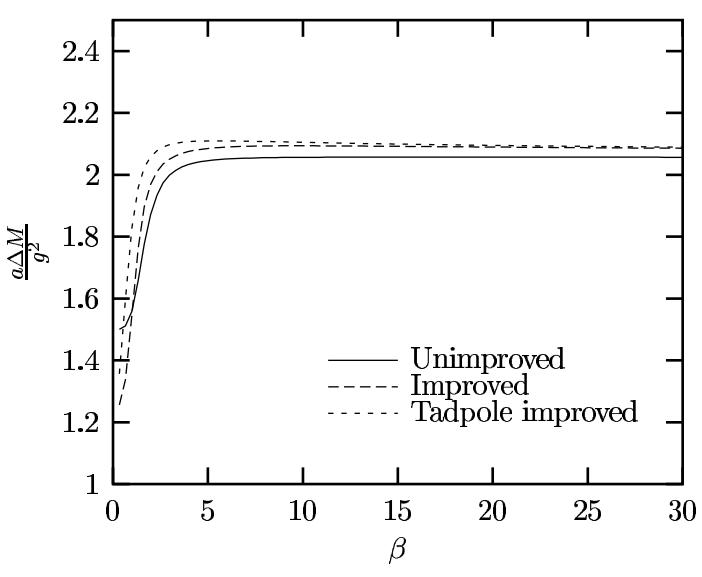

(a) Symmetric SU(2) massgap

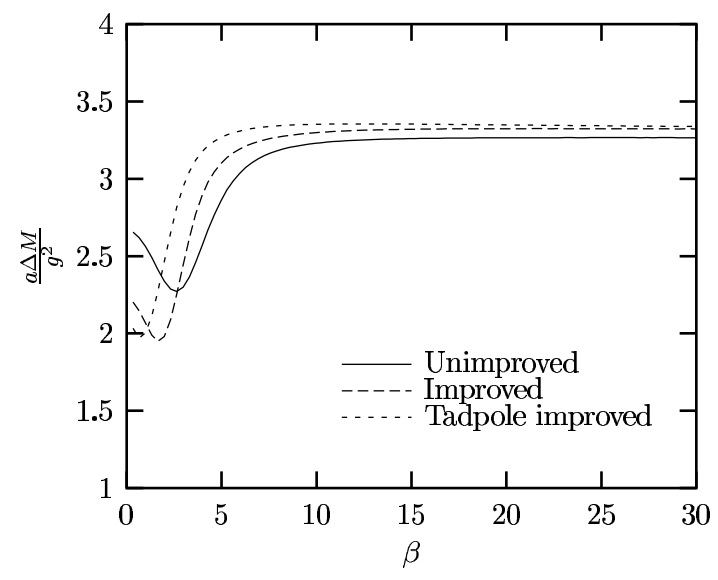

(b) Symmetric SU(3) massgap

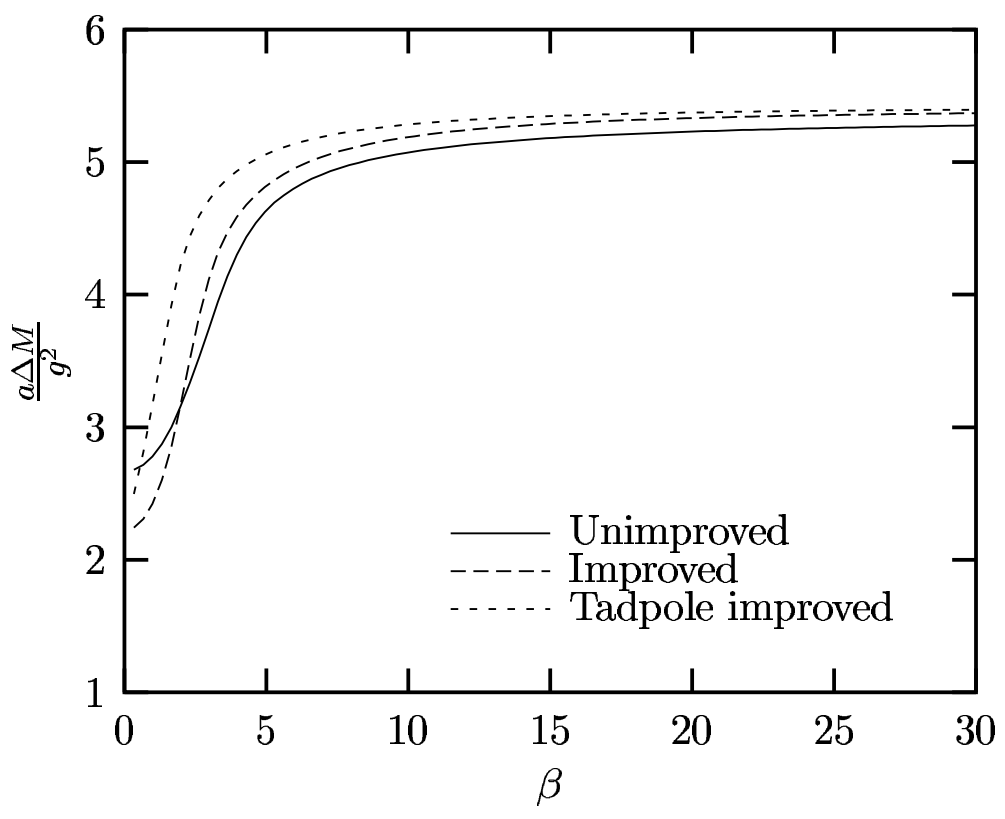

(c) Antisymmetric $\mathrm{SU}(3)$ massgap

Figure 7: $2+1$ dimensional massgaps for $\mathrm{SU}(2)$ and $\mathrm{SU}(3)$ with $L_{\max }=25$. 


\begin{tabular}{llll}
\hline & Unimproved & Improved & Tadpole Improved \\
\hline$\Delta m_{1}^{S}$ & $2.05691 \pm 0.00002$ & $2.0897 \pm 0.0003$ & $2.0965 \pm 0.0006$ \\
$\Delta m_{2}^{S}$ & $3.645 \pm 0.001$ & $3.685 \pm 0.001$ & $3.6953 \pm 0.0009$ \\
$\Delta m_{3}^{S}$ & $4.5202 \pm 0.0004$ & $4.574 \pm 0.004$ & $4.583 \pm 0.004$ \\
$\Delta m_{4}^{S}$ & $5.133 \pm 0.003$ & $5.177 \pm 0.004$ & $5.189 \pm 0.004$ \\
$\Delta m_{5}^{S}$ & $5.867 \pm 0.006$ & $5.932 \pm 0.008$ & $5.943 \pm 0.008$ \\
\hline
\end{tabular}

Table 1: Upper bounds on the lowest lying SU(2) massgaps (in units of $g^{2}$ ) computed with various Hamiltonians in $2+1$ dimensions. The unimproved, improved and tadpole results are calculated in the respective scaling regions $13.5 \leq \beta \leq 30.0,9.9 \leq \beta \leq 30.0$ and $9.25 \leq \beta \leq 30.0$.

\begin{tabular}{llll}
\hline & Unimproved & Improved & Tadpole Improved \\
\hline$\Delta m_{1}^{S}$ & $3.26506 \pm 0.00007$ & $3.3201 \pm 0.0003$ & $3.3350 \pm 0.0008$ \\
$\Delta m_{2}^{S}$ & $6.194 \pm 0.003$ & $6.273 \pm 0.003$ & $6.303 \pm 0.002$ \\
$\Delta m_{3}^{S}$ & $7.396 \pm 0.010$ & $7.536 \pm 0.008$ & $7.575 \pm 0.006$ \\
$\Delta m_{4}^{S}$ & $8.844 \pm 0.007$ & $8.936 \pm 0.008$ & $8.980 \pm 0.006$ \\
$\Delta m_{5}^{S}$ & $9.87 \pm 0.02$ & $10.04 \pm 0.01$ & $10.10 \pm 0.01$ \\
\hline
\end{tabular}

Table 2: Upper bounds on the lowest lying symmetric SU(3) massgaps (in units of $g^{2}$ ) computed with various Hamiltonians in $2+1$ dimensions. The results are calculated in the scaling region $18.5 \leq \beta \leq 50.0$.

The improved SU(2) massgap can be compared to the coupled cluster calculation of Li et al 26]. Their result (in units of $g^{2}$ ), $\Delta m_{1}^{S}=1.59$, is again significantly lower than our result $2.0897 \pm 0.0003$. The difference is attributable to the different choices of ground state used. While our calculation makes use of the simple one plaquette ground state, the coupled cluster calculation of $\mathrm{Li}$ et al uses a more accurate ground state wave function consisting of an exponential of a sum of extended loops.

We can also calculate massgap ratios. For example the antisymmetric-symmetric massgap ratio for $\mathrm{SU}(3)$ is given by

$$
\frac{\Delta m_{1}^{A}}{\Delta m_{1}^{S}}= \begin{cases}1.6173 \pm 0.0008 & \text { Unimproved } \\ 1.6177 \pm 0.0006 & \text { Improved } \\ 1.6182 \pm 0.0005 & \text { Tadpole improved }\end{cases}
$$

These results lie between the unimproved results of Chen et al, 1.6989 [27] and Teper, $1.50 \pm 0.03$ [25]. 


\begin{tabular}{llll}
\hline & Unimproved & Improved & Tadpole Improved \\
\hline$\Delta m_{1}^{A}$ & $5.281 \pm 0.003$ & $5.371 \pm 0.002$ & $5.396 \pm 0.001$ \\
$\Delta m_{2}^{A}$ & $7.831 \pm 0.007$ & $7.947 \pm 0.006$ & $7.986 \pm 0.004$ \\
$\Delta m_{3}^{A}$ & $7.40 \pm 0.01$ & $8.87 \pm 0.01$ & $8.922 \pm 0.009$ \\
$\Delta m_{4}^{A}$ & $10.32 \pm 0.01$ & $10.46 \pm 0.01$ & $10.514 \pm 0.009$ \\
$\Delta m_{5}^{A}$ & $11.06 \pm 0.02$ & $11.27 \pm 0.02$ & $11.33 \pm 0.01$ \\
\hline
\end{tabular}

Table 3: Upper bounds on the lowest lying antisymmetric SU(3) massgaps (in units of $g^{2}$ ) computed with various Hamiltonians in $2+1$ dimensions. The results are calculated in the scaling region $18.5 \leq \beta \leq 50.0$.

\section{Conclusion}

In this paper we have extended the analytic techniques of $2+1$ dimensional Hamiltonian LGT, traditionally used for $\mathrm{SU}(2)$, to the case of SU(3). Impressive scaling is achieved over an extremely wide range of couplings for the lowest energy eigenstates in the symmetric and antisymmetric sectors. Our results use a one plaquette trial state and a basis of rectangular states over which excited state energies are minimised. Such choices allow us to use analytic techniques for both $\mathrm{SU}(2)$ and $\mathrm{SU}(3)$. Our results provide upper bounds on $\mathrm{SU}(2)$ and $\mathrm{SU}(3)$ unimproved, improved and tadpole improved glueball masses all of which are above current estimates. We expect that these upper bounds will lower with the use of more complicated states in the simulation of both the ground state and the excited state. For such choices of states the analytic techniques used here are not as readily applicable.

\section{Acknowledgements}

The work was supported in part by the Australian Research Council.

\section{A Commutation Relations}

In improved Hamiltonian LGT calculations, one encounters matrix elements of the form:

$$
\left\langle\phi_{0}\left|\sum_{\boldsymbol{x}, i} \operatorname{Tr}\left[E_{i}(\boldsymbol{x}) E_{i}(\boldsymbol{x})\right]\right| \phi_{0}\right\rangle \quad \text { and } \quad\left\langle\phi_{0}\left|\sum_{\boldsymbol{x}, i} \operatorname{Tr}\left[E_{i}(\boldsymbol{x}) U_{i}(\boldsymbol{x}) E_{i}(\boldsymbol{x}+a \boldsymbol{i}) U_{i}^{\dagger}(\boldsymbol{x})\right]\right| \phi_{0}\right\rangle .
$$

The first of these is easily handled. One simply writes the electric field operators $E_{i}(\boldsymbol{x})$ in terms of their components with respect to the Gell-Mann matrices $\lambda^{\alpha}, \alpha=1, \ldots, N^{2}-1$,

$$
E_{i}(\boldsymbol{x})=E_{i}^{\alpha}(\boldsymbol{x}) \lambda^{\alpha} .
$$

Making use of the $\mathrm{SU}(N)$ relation

$$
\operatorname{Tr}\left(\lambda^{\alpha} \lambda^{\beta}\right)=\frac{1}{2} \delta_{\alpha \beta},
$$

we have,

$$
\left\langle\phi_{0}\left|\sum_{\boldsymbol{x}, i} \operatorname{Tr}\left[E_{i}(\boldsymbol{x}) E_{i}(\boldsymbol{x})\right]\right| \phi_{0}\right\rangle=\frac{1}{2} \sum_{\boldsymbol{x}, i}\left\langle\phi_{0}\left|E_{i}^{\alpha}(\boldsymbol{x}) E_{i}^{\alpha}(\boldsymbol{x})\right| \phi_{0}\right\rangle
$$


Let the trial state $\left|\phi_{0}\right\rangle$ have the form $\left|\phi_{0}\right\rangle=e^{S}|0\rangle$, where $S$ is a function of link variables and $S^{\dagger}=S$. Then

$$
\sum_{\boldsymbol{x}, i}\left\langle\phi_{0}\left|E_{i}^{\alpha}(\boldsymbol{x}) E_{i}^{\alpha}(\boldsymbol{x})\right| \phi_{0}\right\rangle=-\sum_{\boldsymbol{x}, i}\left\langle 0\left|\left[E_{i}^{\alpha}(\boldsymbol{x}), e^{S}\right]\left[E_{i}^{\alpha}(\boldsymbol{x}), e^{S}\right]\right| 0\right\rangle .
$$

Making use of the Baker-Hausdorff formula we can derive the following result,

$$
\begin{aligned}
e^{S} E_{i}^{\alpha}(\boldsymbol{x}) e^{-S} & =E_{i}^{\alpha}(\boldsymbol{x})-\left[E_{i}^{\alpha}(\boldsymbol{x}), S\right]+\frac{1}{2}\left[\left[E_{i}^{\alpha}(\boldsymbol{x}), S\right], S\right]+\ldots \\
& =E_{i}^{\alpha}(\boldsymbol{x})-\left[E_{i}^{\alpha}(\boldsymbol{x}), S\right] .
\end{aligned}
$$

The last line follows from the fact that $S$ is a function of link variables and the commutation relation:

$$
\left[E_{i}^{\alpha}(\boldsymbol{x}), U_{j}(\boldsymbol{y})\right]=\delta_{i j} \delta_{\boldsymbol{x} \boldsymbol{y}} \lambda^{\alpha} U_{i}(\boldsymbol{x}) .
$$

Some rearrangement of eqn(44) leads to the useful result,

$$
\left[E_{i}^{\alpha}(\boldsymbol{x}), e^{S}\right]=\left[E_{i}^{\alpha}(\boldsymbol{x}), S\right] e^{S}=e^{S}\left[E_{i}^{\alpha}(\boldsymbol{x}), S\right],
$$

which we apply in eqn(43) to obtain,

$$
\begin{aligned}
\sum_{\boldsymbol{x}, i}\left\langle\phi_{0}\left|E_{i}^{\alpha}(\boldsymbol{x}) E_{i}^{\alpha}(\boldsymbol{x})\right| \phi_{0}\right\rangle & =-\sum_{\boldsymbol{x}, i}\left\langle\phi_{0}\left|\left[E_{i}^{\alpha}(\boldsymbol{x}), S\right]\left[E_{i}^{\alpha}(\boldsymbol{x}), S\right]\right| \phi_{0}\right\rangle \\
& =\frac{1}{2} \sum_{\boldsymbol{x}, i}\left\langle\phi_{0}\left|\left[E_{i}^{\alpha}(\boldsymbol{x}),\left[E_{i}^{\alpha}(\boldsymbol{x}), S\right]\right]\right| \phi_{0}\right\rangle .
\end{aligned}
$$

In the last line we have used integration by parts [28].

The second of the matrix elements in eqn(39) is more difficult to handle. For this case it is convenient to expand $E_{i}(\boldsymbol{x}+a \boldsymbol{i})$ in the basis $\tilde{\lambda}_{i}^{\alpha}(\boldsymbol{x}+a \boldsymbol{i})$ :

$$
E_{i}(\boldsymbol{x}+a \boldsymbol{i})=\tilde{\lambda}_{i}^{\alpha}(\boldsymbol{x}+a \boldsymbol{i}) \tilde{E}_{i}^{\alpha}(\boldsymbol{x}+a \boldsymbol{i}),
$$

where,

$$
\tilde{\lambda}_{i}^{\alpha}(\boldsymbol{x}+a \boldsymbol{i})=U_{i}^{\dagger}(\boldsymbol{x}) \lambda^{\alpha} U_{i}(\boldsymbol{x}) .
$$

We now derive the commutation relations between $\tilde{E}_{i}^{\alpha}(\boldsymbol{x})$ and $U_{j}(\boldsymbol{y})$. We start with the analogous relation to eqn(41),

$$
\operatorname{Tr}\left[\tilde{\lambda}_{i}^{\alpha}(\boldsymbol{x}) \tilde{\lambda}_{i}^{\beta}(\boldsymbol{x})\right]=\frac{1}{2} \delta_{\alpha \beta},
$$

which follows trivially from eqn(49). This relation allows us to invert eqn(48) and write $\tilde{E}^{\alpha}$ in terms of $E^{\alpha}$

$$
\begin{aligned}
\tilde{E}_{i}^{\alpha}(\boldsymbol{x}) & =2 \operatorname{Tr}\left[E_{i}(\boldsymbol{x}) \tilde{\lambda}_{i}^{\alpha}(x)\right] \\
& =2 \operatorname{Tr}\left[\tilde{\lambda}_{i}^{\alpha}(\boldsymbol{x}) \lambda^{\beta}\right] E_{i}^{\beta}(\boldsymbol{x}) .
\end{aligned}
$$


We can form a similar relation between $\tilde{\lambda}^{\alpha}$ and $\lambda^{\alpha}$ as follows. Since $\tilde{\lambda}_{i}^{\alpha}(\boldsymbol{x}) \in \operatorname{SU}(N)$, we can expand in the Gell-Mann basis as follows,

$$
\tilde{\lambda}_{i}^{\alpha}(\boldsymbol{x})=c_{i}^{\alpha \gamma}(\boldsymbol{x}) \lambda^{\gamma}
$$

Here $c_{i}^{\alpha \beta}(x)$ are constants which we now determine. Multiplying eqn(52) throughout by $\lambda^{\beta}$, tracing and making use of eqn(41) gives

$$
c_{i}^{\alpha \beta}(\boldsymbol{x})=2 \operatorname{Tr}\left[\tilde{\lambda}_{i}^{\alpha}(\boldsymbol{x}) \lambda^{\beta}\right] .
$$

From eqn(51) we see that $\tilde{E}_{i}^{\alpha}(\boldsymbol{x})=c_{i}^{\alpha \beta}(\boldsymbol{x}) E_{i}^{\beta}(\boldsymbol{x})$. The commutation relations follow immediately,

$$
\begin{aligned}
{\left[\tilde{E}_{i}^{\alpha}(\boldsymbol{x}), U_{j}(\boldsymbol{y})\right] } & =c_{i}^{\alpha \beta}(\boldsymbol{x})\left[E_{i}^{\beta}(\boldsymbol{x}), U_{j}(\boldsymbol{y})\right]=c_{i}^{\alpha \beta}(\boldsymbol{x}) \lambda^{\beta} \delta_{i j} \delta_{\boldsymbol{x} \boldsymbol{y}} U_{i}(\boldsymbol{x}) \\
& =\delta_{i j} \delta_{\boldsymbol{x} \boldsymbol{y}} \tilde{\lambda}_{i}^{\alpha}(\boldsymbol{x}) U_{i}(\boldsymbol{x})
\end{aligned}
$$

These commutation relations enable the simplification the second of the matrix elements in eqn(39) using the basis defined in eqn(48). Making use of eqns (51) and (54) leads to the following simplification:

$$
\left\langle\phi_{0}\left|\sum_{\boldsymbol{x}, i} \operatorname{Tr}\left[E_{i}(\boldsymbol{x}) U_{i}(\boldsymbol{x}) E_{i}(\boldsymbol{x}+a \boldsymbol{i}) U_{i}^{\dagger}(\boldsymbol{x})\right]\right| \phi_{0}\right\rangle=\frac{1}{4} \sum_{\boldsymbol{x}, i}\left\langle\phi_{0}\right|\left[E_{i}^{\alpha}(\boldsymbol{x}),\left[\tilde{E}_{i}^{\alpha}(\boldsymbol{x}+a \boldsymbol{i}), S\right]\left|\phi_{0}\right\rangle .\right.
$$

In order to calculate such matrix elements the following results are useful

$$
\begin{gathered}
{\left[E_{i}^{\alpha}(\boldsymbol{x}),\left[\tilde{E}_{i}^{\alpha}(\boldsymbol{x}+a \boldsymbol{i}), U_{i}(\boldsymbol{x}) U_{i}(\boldsymbol{x}+a \boldsymbol{i})\right]\right]=\frac{N^{2}-1}{2 N} U_{i}(\boldsymbol{x}) U_{i}(\boldsymbol{x}+a \boldsymbol{i}),} \\
{\left[E_{i}^{\alpha}(\boldsymbol{x}),\left\{U_{i}(\boldsymbol{x})\right\}_{A B}\right]\left[\tilde{E}_{i}^{\alpha}(\boldsymbol{x}+a \boldsymbol{i}),\left\{U_{i}(\boldsymbol{x}+a \boldsymbol{i})\right\}_{C D}\right]=} \\
\frac{1}{2} \delta_{B C}\left\{U_{i}(\boldsymbol{x})\right\}_{A B^{\prime}}\left\{U_{i}(\boldsymbol{x}+a \boldsymbol{i})\right\}_{B^{\prime} D}-\frac{1}{2 N}\left\{U_{i}(\boldsymbol{x})\right\}_{A B}\left\{U_{i}(\boldsymbol{x}+a \boldsymbol{i})\right\}_{C D}, \\
{\left[E_{i}^{\alpha}(\boldsymbol{x}),\left\{U_{i}^{\dagger}(\boldsymbol{x})\right\}_{A B}\right]\left[\tilde{E}_{i}^{\alpha}(\boldsymbol{x}+a \boldsymbol{i}),\left\{U_{i}(\boldsymbol{x}+a \boldsymbol{i})\right\}_{C D}\right]=} \\
-\frac{1}{2}\left\{U_{i}^{\dagger}(\boldsymbol{x})\right\}_{C B}\left\{U_{i}(\boldsymbol{x}+a \boldsymbol{i})\right\}_{A D}+\frac{1}{2 N}\left\{U_{i}^{\dagger}(\boldsymbol{x})\right\}_{A B}\left\{U_{i}(\boldsymbol{x}+a \boldsymbol{i})\right\}_{C D}, \\
{\left[E_{i}^{\alpha}(\boldsymbol{x}),\left\{U_{i}(\boldsymbol{x})\right\}_{A B}\right]\left[\tilde{E}_{i}^{\alpha}(\boldsymbol{x}+a \boldsymbol{i}),\left\{U_{i}^{\dagger}(\boldsymbol{x}+a \boldsymbol{i})\right\}_{C D}\right]=} \\
-\frac{1}{2}\left\{U_{i}(\boldsymbol{x})\right\}_{C B}\left\{U_{i}^{\dagger}(\boldsymbol{x}+a \boldsymbol{i})\right\}_{A D}+\frac{1}{2 N}\left\{U_{i}(\boldsymbol{x})\right\}_{A B}\left\{U_{i}^{\dagger}(\boldsymbol{x}+a \boldsymbol{i})\right\}_{C D} .
\end{gathered}
$$

Here $\{X\}_{A B}$ denote the colour indices of the matrix $X$ and an implicit sum over repeated colour indices is understood. These results follow simply from the commutation relations of eqns (45) and (54), and the following $\mathrm{SU}(N)$ formula:

$$
\lambda_{A B}^{\alpha} \lambda_{C D}^{\alpha}=\frac{1}{2} \delta_{A D} \delta_{B C}-\frac{1}{2 N} \delta_{A B} \delta_{C D}
$$

\section{References}

[1] K. G. Wilson, Phys. Rev. D10, 2445 (1974). 
[2] J. B. Kogut and L. Susskind, Phys. Rev. D11, 395 (1975).

[3] L. C. Hollenberg, Phys. Rev. D 47, 1640 (1993).

[4] L. C. Hollenberg, Phys. Rev. D 50, 6917 (1994).

[5] J. A. McIntosh and L. C. Hollenberg, Z. Phys. C 76, 175 (1997).

[6] M. P. Wilson and L. C. Hollenberg, Austral. J. Phys. 51, 35 (1998).

[7] F. Coester, Nucl. Phys. 7, 421 (1958).

[8] F. Coester and H. G. Kümmel, Nucl. Phys. 17, 477 (1960).

[9] N. E. Ligterink, N. R. Walet and R. F. Bishop, Nucl. Phys. A663, 983 (2000).

[10] B. H. McKellar, C. R. Leonard and L. C. Hollenberg, Int. J. Mod. Phys. B14, 2023 (2000).

[11] H. G. Kümmel, K. H. Luhrmann and J. G. Zabolitsky, Phys. Reports 36, 1 (1978).

[12] E. B. Gregory, S. H. Guo, H. Kroger and X. Q. Luo, Phys. Rev. D62, 054508 (2000).

[13] G. P. Lepage, arXiv:hep-lat/9607076.

[14] X. Q. Luo, S. H. Guo, H. Kroger and D. Schutte, Phys. Rev. D 59, 034503 (1999).

[15] J. Carlsson and B. H. McKellar, Phys. Rev. D64, 094503 (2001).

[16] J. Greensite, Phys. Lett. B 191, 431 (1987).

[17] G. G. Batrouni and M. B. Halpern, Phys. Rev. D 30, 1782 (1984).

[18] K. E. Eriksson, N. Svartholm and B. S. Skagerstam, J. Math. Phys. 22, 2276 (1981).

[19] M. Creutz, Cambridge, Uk: Univ. Pr. (1983) 169 P. (Cambridge Monographs On Mathematical Physics).

[20] H. Arisue, Prog. Theor. Phys. 84, 951 (1990).

[21] S. P. Tonkin, Nucl. Phys. B 292, 573 (1987).

[22] I. Bars, J. Math. Phys. 21, 2678 (1980).

[23] X. Q. Luo and Q. Z. Chen, Mod. Phys. Lett. A 11, 2435 (1996).

[24] S. Samuel, Phys. Rev. D 55, 4189 (1997).

[25] M. J. Teper, Phys. Rev. D 59, 014512 (1999).

[26] J. Li, S. Guo and X. Q. Luo, Commun. Theor. Phys. 34, 301 (2000).

[27] Q. Z. Chen, X. Q. Luo, S. H. Guo and X. Y. Fang, Phys. Lett. B 348, 560 (1995).

[28] H. Arisue, M. Kato and T. Fujiwara, Prog. Theor. Phys. 70, 229 (1983). 